\title{
Application of SAX in temperature monitoring of friction welding
}

\section{Du Kun}

\begin{abstract}
In this work,we introduce the new way to find problem in frictionwelding.SAX is a new way to find discords. Symbolic aggregate approimation(SAX representation) is a time series dimensionality reduction technique which benefits from its brief represention in dimensionality reduction and high-performance lower bound distance algorithm. In friction welding process, temperature is an important index to judge whether the machining is normal or not.SAX method provides a fast and efficient way for temperature detection, which can quickly find the abnormal region of temperature data.This method can quickly detect the location of the abnormal area and find the cause of the exception.
\end{abstract}

\section{Index Terms - Detection, Frictionweld, SAX, Temperatrue}

\section{INTRODUCTION}

Frictionwelding is a kind of pressure welding, it uses two welding contacted surfaces rotating relatively to generat the heat.Then it conme to the state of thermoplastic .After the quickly die forging, it completes the welding .It is a solid-phase bonding method, which is different from other welding methods.Since ancient times, people have known the phenomenon that friction generates heat. Until modern times, this phenomenon has not been applied to industrial production. The origin of friction applied to welding, namely friction welding, can be traced back to the United States in 1891.Subsequently, Germany, Britain, Japan, the former Soviet union and other countries have also carried out friction welding research ${ }^{[1]}$.

Monitoring technology is a comprehensive technology that integrates sensor technology, computer technology and information technology $y^{[2]}$. As a key technology of modern integrated manufacturing system, it is widely used in the process of modern industrial production.A good monitoring system can effectively obtain important information of the production process, controlig the production process and improving the quality of products.In the process of industrial production, temperature is an important and common physical parameter, and temperature monitoring technology has more and more important research significance and value ${ }^{[3]}$.

As a traditional process, the heating of metal workpiece is the key process to realize heat treatment and pressure machining. The heating process of metal workpiece is usually realized in industrial kilns. In order to prevent the workpiece from heating unevenly, stress cracks and abnormal microstructure, the temperature of the workpiece can be monitored in real time, and the heating quality and efficiency can be guaranteed by controlling the heating speed, heating uniformity and real-time temperature of the workpiece.At present, scholars study and adopt the workpiece processing

Du Kun is a Master student of Jiangsu University of Science and Technology. The main research directions was Anomaly monitoring technique monitoring method, and indirectly measure the workpiece temperature by selecting the temperature of each characteristic point in the furnace, so as to monitor the workpiece heating speed, heating uniformity and real-time temperature.

\section{RESEARCH METHODS}

In the friction welding process, the temperature change can largely reflect the quality of the processing process, there are many factors can lead to the abnormal temperature in the processing process.

Friction welding is a thermally coupled process, which is accompanied by metal plastic deformation, metal plastic flow, friction heat conduction and energy conversion.In each stage of welding, the temperature must reach the corresponding value in order to make the normal processing process. Therefore, the monitoring of temperature changes in the processing process can find out the location and cause of problems in the production process in time.

In this paper, the temperature changes in the friction welding process of no. 45 steel bar were collected, and the temperature data and change curves in the friction welding process under ideal conditions were simulated by finite element analysis.By comparing the collected temperature data with the ideal temperature data, the abnormal temperature data can be found, and the possible problems in the whole friction welding process can be found out.

\section{SAX REPRESENTATION}

SAX is based on PAA (piecewise approximation) theory, it unites temporal characteristic of normal distribution.It is a representation of time series symbolization conversion, and can not only effectively reduce the temporal dimensionb but also can support real-time data streaming transformation, such as clustering, classification and anomaly detection algorithm has a good performance ${ }^{[4]}$. The process of SAX representation of time series is as follows:

1) the normal standardized formula is adopted to process the time series, and the time series data are transformed to conform to the standard normal distribution.

2) given the reference time series of length $n, C=C_{1}, C_{2}$ $, \ldots, \mathrm{C}_{\mathrm{n}}$, which is processed by PAA dimensionality reduction, use dimension vector $\mathrm{C}=\mathrm{C}_{1}, \mathrm{C}_{2}, \ldots$, the $\mathrm{Cw}$ says, where $C_{i}$ is derived from equation (1):

$$
\overline{c_{i}}=\frac{w}{n} \sum_{j=\frac{\sum_{n(i-1)}^{\frac{n}{\omega} i}}{\omega}+1} c_{i}
$$

3)PAA (Piecewise Aggregate Approximation) is the sequence of dimension reduction. 
Aaccording to the distribution features of sequence, we use the nature of the standard normal distribution probability density function to set a number of point that divide the domain into the numerical sequence of probability distribution of multiple regions, discretized and the symboled the sequence ${ }^{[5]}$.

\section{IV. .ESTABLISHMENT OF FRICTION WELDING PROCESS MODEL}

In the process of welding, the geometric shapes of the two workpieces to be welded are the same. The specimens are 45 steel bars with a diameter of $10 \mathrm{~mm}$ and a length of $75 \mathrm{~mm}$, as shown in Figure 1.

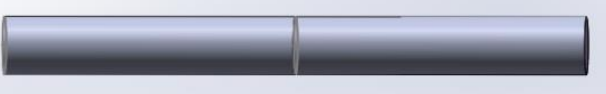

Figure. 1 welding specimen model diagram

Elastic deformation is far less than the plastic deformation in the welding process, and the temperature is very high[6].So, the welding area closed to the melting temperature of the material where material in large plastic deformation.It will lead to deformation of the material with heat conduction problems, so the calculation method is based on the analysis of simulated in just - viscoplastic finite element method (fem).We regard of the elastic deformation of the elastic deformation which is far less than the plastic deformation in the welding process.

By analyzing the friction welding principle, the influence of welding parameters on the welding mechanism, and combining the analysis of the weldability and welding difficulty of no.45 steel, the welding process is finally determined as follows:

1). Spindle speed is $3000 \mathrm{r} / \mathrm{min}$

2 ). The primary friction time is $8 \mathrm{~s}$, and the secondary friction time is $6 \mathrm{~s}$.

$3)$. Friction pressure: according to the working principle of friction welder and a large number of tests, the size of the first friction pressure is about $1 / 2$ of the second friction pressure. Set the first-stage friction pressure as $7 \mathrm{k} \mathrm{N}$ and the second-stage friction pressure as $15 \mathrm{k} \mathrm{N}$ [7].

4). Upsetting time: is the time when upsetting pressure is applied. The test is set as $3 \mathrm{~s}$ upsetting pressure with $30 \mathrm{KN}$.

By using Ansys software, the specimens obtained in the friction welding process are shown in Figure2.

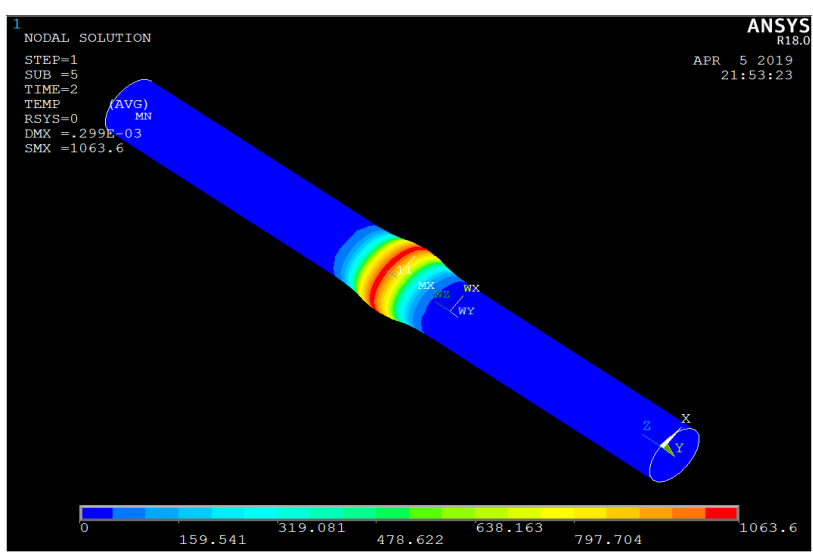

Figure. 2 thermal analysis diagram of specimens in the welding process

\section{TEMPERATURE CALCULATION RESULT}

The calculated result model is divided along the friction surface to facilitate the calculation of the temperature at different times and the distribution of the temperature on the welding surface. Figure 3, Figure 4 and Figure 5 show the temperature isoline distribution of the friction surface on the steel side of the welded joint at different times in the three stages of primary friction, secondary friction and upset.Figure 6 shows the change of temperature with time at the radius $2 / 3$ of the friction surface from the center of the circle.

Figure 3 shows the temperature field distribution of the welded joint at the end of the first friction stage.

Figure 4 shows the temperature field distribution of the welded joint at the end of the second friction stage.Is high temperature area, and the friction on the surface of the high temperature area becomes uniform, the highest temperature of $1100^{\circ} \mathrm{C}$

Figure 5 shows the temperature field distribution of the welded joints at the end of the top forging stage.High temperature area is relatively smaller secondary friction stage, and the temperature drop, the highest temperature reached $800{ }^{\circ} \mathrm{C}$, welding surface rendering large temperature gradient.

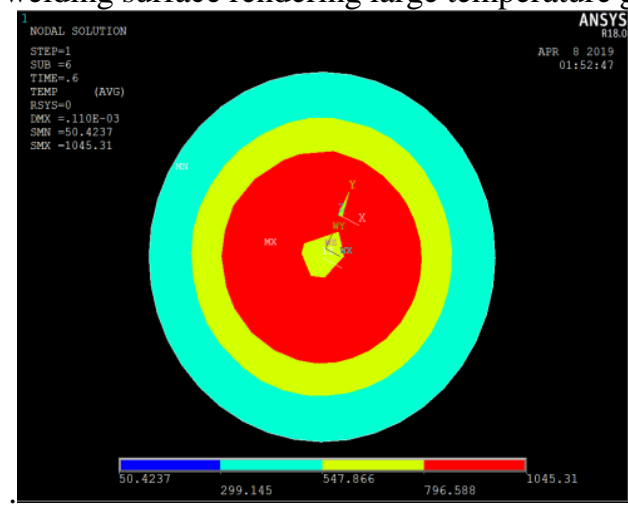

Figure 3 friction surface temperature distribution at $t=8 \mathrm{~s}$

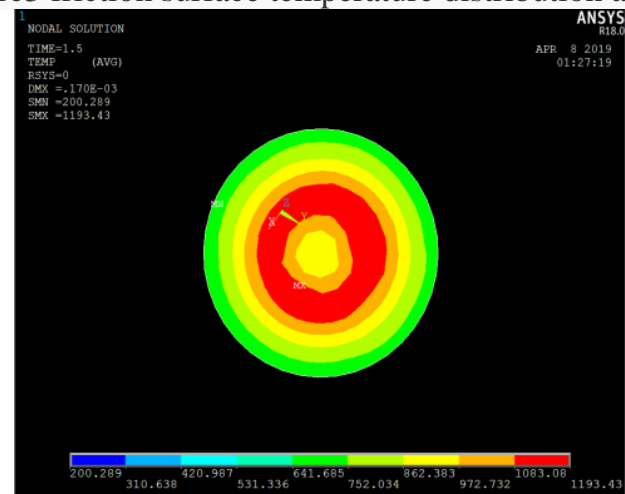

Figure 4 temperature distribution of friction surface at $\mathrm{t}=14 \mathrm{~s}$

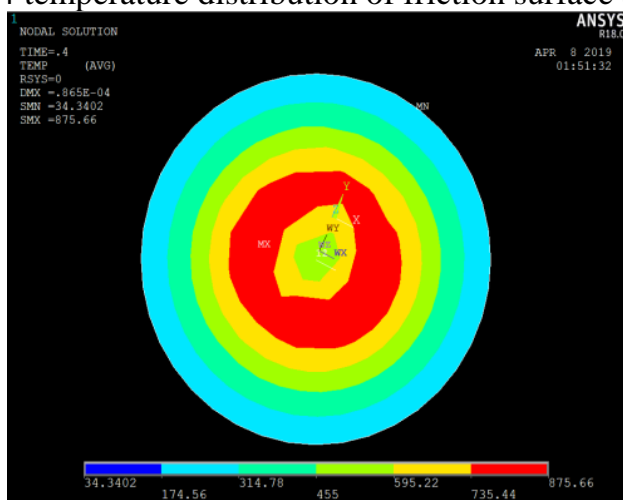

Figure 5 temperature distribution of friction surface at $\mathrm{t}=17 \mathrm{~s}$ 
Figure 6 shows the change of temperature with time at the radius $2 / 3$ of the friction surface from the center of the circle. The optimum result of temperature curve is determined through many experiments and simulations. We can find out from the diagram that the level of friction stage begins, $1 \mathrm{~s}$ temperature change is not obvious. At the time of 1.5 $\mathrm{s}$,friction surface temperature had obvious changes, warming faster, continued into the secondary friction stage.It reached to the highest at $14 \mathrm{~s}$. The temperature at this time is about $1100{ }^{\circ} \mathrm{C}$.In the upended stage, the temperature drops faster, until the end of welding.

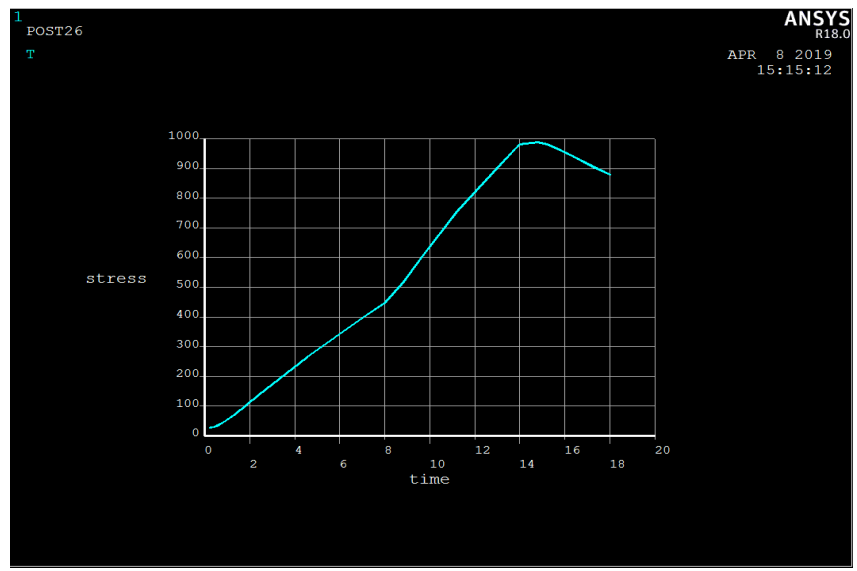

Figure 6 curve of temperature change with time at the radius $2 / 3$ of the friction surface from the center of the circle

\section{RESULT}

The anomaly detection method based on SAX symbolic representation is described as follows:

1) to transform the original time sequence for PAA (Piecewise Aggregate Approximation) said.First, the nonoverlapping fixed-size sliding window is used to obtain the sequence of equal length.

2) then it is converted into SAX symbolic representation.On the basis of PAA representation of time series, the numerical value of time series is further discretized and converted into the symbolic representation of SAX.In order to consider the efficiency of the subsequent algorithm, the symbol of equal probability should be generated as much as possible in the process of discretization ${ }^{[8]}$.

3) detect abnormal events.Each sub-sequence of the diachronic sequence is iterated through a two-level loop.The non-self-matching distance of each sub-sequence is calculated $^{[9]}$.

We firstly obtained the difference value between the temperature data collected in the experiment and the ideal temperature data.

Then the deviation between the temperature data at each time and the ideal state can be obtained.

Thirdly, the region with the largest anomaly in the data is found through SAX method, and the position of the region with the largest anomaly in the whole friction welding process can be analyzed.we can judge whether it is in the first friction stage, the second friction stage, or the forging stage.At the same time, it can judge whether the production process is normal according to the deviation degree of its data ${ }^{[10]}$. If the maximum abnormal area is beyond the reasonable range, the reasons for the abnormal can be found in the production stage.

\section{REFERENCES:}

[1] WEN D , ZENG H, KE L • PID paramenter optimization of the resistance-heated furnace control system based on genetic algorithm [J ] .Electrical Automation,2008,30( 4) : 6-8 .

[2] Kumar, N., Lolla N., Keogh, E., Lonardi, S, Ratanamahatana, C., \& Li, W. (2005). Time-series Bitmaps: A Practical Visualization Tool for working with Large Time Series Databases. SIAM Data Mining Conference.

[3] Xu Xiaoling,Wu Wei,Xu Yuanze.The research of radial friction welding[J].Welding in the World,2005,49 (1/2): 12-15.

[4] Ratanamahatana, C. \& Keogh, E. (2004). Making Time-series Classification More Accurate Using Learned Constraints. In proceedings of the 4th SIAM International Conference on Data Mining.

[5] ROBERTSJJ,BESTBED,DUNNDC,etal.Marine geospatial ecology tools: an integrated framework for ecological geoprocessing with ArcGIS,Python, R,Matlab,and C++ [J].Environmental Modelling and Software,2010,25( 10) : 1197-1207.

[6] Ruzzo W.L, \& Tompa M. (1999). A linear time algorithm for finding all maximal scoring subsequences. In Proc Int Conf Intell Syst Mol Biol.; pp 234-41.

[7] LUO H · Study notes of Matlab GUI design [ $M$ ] 2nded.Beijing:Beijing University of Aeronautics and Astronautics Press,2011: 87-673.

[8] LIU X,LI J.Design of a multi-axis control system based on MODBUS protocol[J] Machinery Design \&Manufacture,2013( 2) : 1-3.

[9] Kallee S W,Thomas W M , Burling P M , et al.Friction stir welding of aluminum extrusions in the transport industry[C]//International Conference on Welding and ManufactureTechnology.Vienna: [s.n],2000.

[10] Ho Slung Jeon,Joni-Rae.Inertia friction welding processanalysis and mechanical properties evaluation of large rotorshaft in marine turbot charger $[\mathrm{J}] \cdot$.Welding Journal,2010,11( 1) : $83-88$ 